\title{
Decrease of the electric field penetration into the ionosphere due to low conductivity at the near ground atmospheric layer
}

\author{
M. Ampferer ${ }^{1}$, V. V. Denisenko ${ }^{2,3}$, W. Hausleitner ${ }^{4}$, S. Krauss ${ }^{4}$, G. Stangl ${ }^{4,5}$, M. Y. Boudjada ${ }^{4}$, and H. K. Biernat ${ }^{1,4,6}$ \\ ${ }^{1}$ Institute of Physics, Department of Geophysics, Astrophysics and Meteorology, Karl-Franzens-University Graz, Austria \\ ${ }^{2}$ Institute of Computational Modelling, Russian Academy of Sciences, Siberian Branch, Krasnoyarsk, Russia \\ ${ }^{3}$ Siberian Federal University, Krasnoyarsk, Russia \\ ${ }^{4}$ Space Research Institute, Austrian Academy of Sciences, Graz, Austria \\ ${ }^{5}$ Federal Office of Metrology and Surveying, Vienna, Austria \\ ${ }^{6}$ Institute of Physics, Department of Theoretical Physics, Karl-Franzens-University Graz, Austria
}

Received: 12 November 2009 - Revised: 3 March 2010 - Accepted: 4 March 2010 - Published: 16 March 2010

\begin{abstract}
It is well known that lithospheric electromagnetic emissions are generated before earthquakes occurrence. In our study, we consider the physical penetration mechanism of the electric field from the Earth's surface, through the atmosphere-ionosphere layers, and until its detection in space by satellites. A simplified approach is investigated using the electric conductivity equation, i.e., $\nabla(\hat{\sigma} \cdot \nabla \Phi)=0$ in the case of a vertical inclination of the geomagnetic field lines. Particular interest is given to the conductivity profile near the ground and the electric field distribution at the Earth's surface. Our results are discussed and compared to the models of Pulinets et al. (2003) and Denisenko et al. (2008). It is shown that the near ground atmospheric layer with low conductivity decreases the electric field penetration into the ionosphere. The model calculations have demonstrated that the electric field of lithospheric origin is too weak to be observed at satellite altitudes.
\end{abstract}

Keywords. Ionosphere (Electric fields and currents; Ionosphere-atmosphere interactions) - Meteorology and atmospheric dynamics (Atmospheric electricity)

\section{Introduction}

First unusual disturbances of the electrical conditions in the atmosphere has been reported prior to large earthquakes by Kondo (1968). The systematic research started with Gokhberg et al. (1983) and several authors investigated

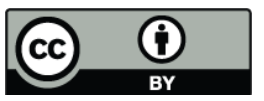

Correspondence to: $\mathrm{H}$. K. Biernat (helfried.biernat@oeaw.ac.at) the behavior of the atmosphere and the ionosphere due to strong earthquakes (Kelley et al., 1985; Kingsley, 1989; Pogorel'tsev, 1989; Depuev and Zelenova, 1996; Ruzhin and Depueva, 1996).

Seismic precursory phenomena are generally related to the formation of micro-cracks in the lithosphere days to weeks before the event, which increase in their number density and finally end in the earthquake itself (Molchanov and Hayakawa, 1995). Several models have been proposed to explain the main physical mechanisms and the corresponding effects starting from the ground up to the ionosphere (Gokhberg et al., 1985; Pulinets et al., 2003; Sorokin et al., 2001; Molchanov et al., 2004). Characteristic variations were reported in the critical frequency $f o \mathrm{~F} 2$ (Silina et al., 2001), the total electron content (Liu et al., 2004), the ion temperature (Sharma et al., 2006), or the local ion and electron density (Berthelier et al., 2006). Detailed reviews can be found in Molchanov and Hayakawa (2008) and Pulinets and Boyarchuk (2004).

\subsection{Electric field penetration into the ionosphere}

In the frame of ionospheric precursors, it is important to know what kind of effect could be observable from space to maintain proper satellite missions. In this connection it is essential to understand how electric fields from lithospheric origin penetrate into higher altitudes of the atmosphere. The first theoretical investigations of the penetration of an electrostatic field from the lithosphere into the ionosphere were done by Park and Dejnakarintra (1973). These authors analytically studied the penetration of thundercloud electric fields into the ionosphere and the magnetosphere. For this

Published by Copernicus Publications on behalf of the European Geosciences Union. 
approach they assumed straight and vertical geomagnetic field lines as well as a vertical electric conductivity profile of piecewise exponential functions. Several authors (Kim et al., 1994; Pulinets et al., 1998; Grimalsky et al., 2003; Denisenko et al., 2008) adapted their theoretical works on the penetration of electric fields into the ionosphere.

There is a contradiction between the results of these models. The model by Pulinets et al. (1998) and further modifications of that model reviewed in Pulinets et al. (2003) predict an electric field of about $1 \mathrm{mV} \mathrm{m}^{-1}$ in the ionosphere when a $100 \mathrm{~V} \mathrm{~m}^{-1}$ vertical electric field appears near ground. The model investigated by Denisenko et al. (2008) predicts in the same case much less fields, $10 \mu \mathrm{V} \mathrm{m}^{-1}$ in the night time ionosphere and $0.1 \mu \mathrm{V} \mathrm{m}^{-1}$ at day time.

The main difference between these models is related to the boundary condition in the upper atmosphere. It excludes conductivity of the ionosphere in the model of $\mathrm{Pu}$ linets et al. (1998, 2000) and Kim et al. (1996) and includes the integral conductivity of the ionosphere in the model by Denisenko et al. (2008)

Denisenko et al. (2008) showed that the field penetration is inverse proportional to the value of the ionospheric Pedersen conductance, which is about 100 times less during nighttime than during day-time conditions. They concluded that the electric field penetration is damped and it is not possible to detect a seismic precursor signal at ionospheric heights, because larger electric fields of other nature always exist in the ionosphere. There was a strict simplification in that model. An exponential function was used to approximate the height distribution of the atmospheric conductivity as shown in Fig. 1.

In this paper, we are interested in the behavior of the electric field penetration, when we use a better approximation for the real profile of the electric conductivity. A second advancement is done for the electric field on the ground where we use a bipolar distribution, as well as it is done in the model of Kim et al. (1996).

\section{Basic equations}

It is possible to use a steady state model for a conductor with a conductivity $\sigma$ when the typical time of the process is much larger than the charge relaxation time $\tau=\varepsilon_{0} / \sigma$ (Molchanov and Hayakawa, 2008). In the atmosphere $\sigma>10^{-14} \mathrm{~S} \mathrm{~m}^{-1}$. Hence $\tau<10^{3} \mathrm{~s}$, and the steady state model is adequate for processes longer than one hour. The basic equations for the atmospheric electric field $\mathbf{E}$ are the Faraday law, the charge conservation law, and Ohm's law,

$$
\begin{aligned}
& \nabla \times \mathbf{E}=0, \\
& \nabla \cdot \mathbf{j}=0, \\
& \mathbf{j}=\sigma \mathbf{E},
\end{aligned}
$$

where $\mathbf{j}$ is the current density. In the atmosphere, the conductivity is approximately isotropic up to $z_{\mathrm{up}}=80 \mathrm{~km}$ which is the upper altitude range of the obtained model. Because of (Eq. 1) the electric potential $\Phi$ can be introduced so that

$\mathbf{E}=-\nabla \Phi$

Then the system of the equations (Eqs. 1-3) is reduced to the equation

$-\nabla \cdot(\sigma \cdot \nabla \Phi)=0$.

\section{Conductivity}

It can be seen from (Eq. 5) that the electric conductivity $\sigma$ takes a major part in our investigations. In the model (Denisenko et al., 2008) the electric conductivity was presented with an exponential function of the height $z$

$\sigma(z)=\bar{\sigma} \exp (z / h)$,

where the conductivity only depends on an initial value $\bar{\sigma}$ and on the scale height $h$.

For a better approximation to a real profile of the atmospheric conductivity, we separate the height distribution into three layers at altitudes of $z_{\text {low }}=2.8 \mathrm{~km}$ and $z_{\text {mid }}=66 \mathrm{~km}$. In accordance to measurements reported by Molchanov and Hayakawa (2008), we assume that the electric conductivity is about 30 times less near the ground then it would be if its height dependence is extrapolated from the rest of the atmosphere (Fig. 1). The value at the bottom of the ionosphere is $10^{-7} \mathrm{~S} \mathrm{~m}^{-1}$ in this model as it is in the approximated profile while it was $6 \times 10^{-8} \mathrm{~S} \mathrm{~m}^{-1}$ in the simplified approximation (Eq. 6). The values for the actual three-layer model are given by

$$
\begin{aligned}
& \bar{\sigma}_{1}=10^{-14} \mathrm{~S} / \mathrm{m}, \quad h_{1}=0.81 \mathrm{~km}, \quad 0 \leq z<z_{\text {low }}, \\
& \bar{\sigma}_{2}=3 \times 10^{-13} \mathrm{~S} / \mathrm{m}, \quad h_{2}=7.5 \mathrm{~km}, \quad z_{\text {low }} \leq z<z_{\text {mid }}, \\
& \bar{\sigma}_{3}=1.4 \times 10^{-9} \mathrm{~S} / \mathrm{m}, h_{3}=3.3 \mathrm{~km}, z_{\text {mid }} \leq z \leq z_{\text {up }},
\end{aligned}
$$

and the formula Eq. (6) is modified as

$$
\begin{aligned}
& \sigma(z)=\bar{\sigma}_{2} \exp \left(\left(z-z_{\text {low }}\right) / h_{2}\right), \\
& \sigma(z)=\bar{\sigma}_{3} \exp \left(\left(z-z_{\text {mid }}\right) / h_{3}\right),
\end{aligned}
$$

in the second and the third layer, respectively.

\section{Model geometry}

The geometry of the model is chosen in that way, that the origin of the coordinate system is located exactly at the epicenter, the $\mathrm{y}$-axis is directed along the fault, and the $\mathrm{x}$-axis is directed perpendicular to the fault. The $\mathrm{z}$-axis is directed upward, from the ground towards the ionosphere. The distribution only depends on the distance perpendicular to the tectonic fault $x$, but along the fault $y$ the field is considered to be not variable. This simple approach is consistent with 


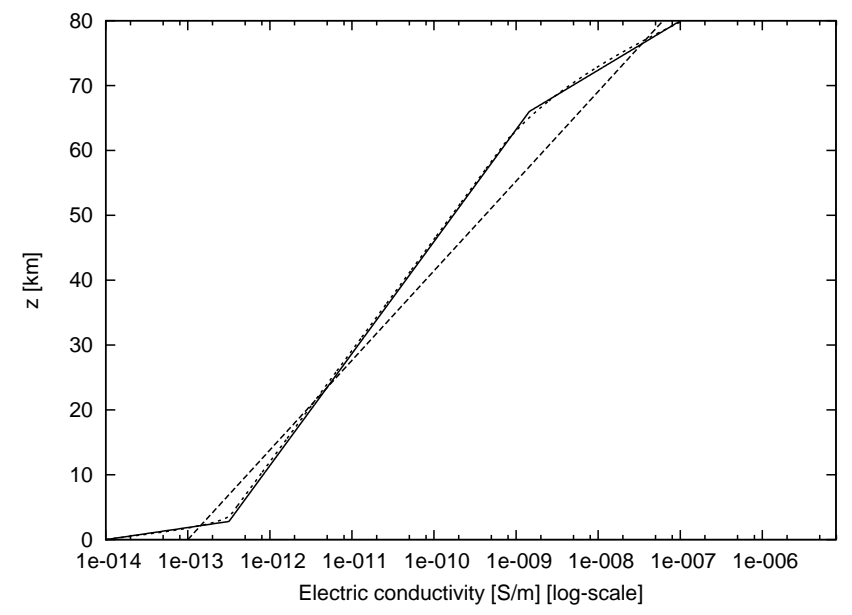

Fig. 1. Conductivity profile of the atmosphere up to an altitude of $80 \mathrm{~km}$. The curves indicate the actual three-layer model (solid line), the model of Denisenko et al. (2008) (dashed line), and the empirical model reported by Molchanov and Hayakawa (2008) (dotted line).

earlier works (Pulinets et al., 1998; Denisenko et al., 2008). We analyze the two-dimensional model in which no parameter depends on $y$. In such a model, Eq. (5) has the form

$-\frac{\partial}{\partial x}\left[\sigma(z) \frac{\partial}{\partial x} \Phi(x, z)\right]-\frac{\partial}{\partial z}\left[\sigma(z) \frac{\partial}{\partial z} \Phi(x, z)\right]=0$.

The general solution for this equation can be obtained by separation of the variables in the three different altitude ranges $0 \leq z<z_{\text {low }}, z_{\text {low }} \leq z<z_{\text {mid }}$ and $z_{\text {mid }} \leq z \leq z_{\text {up. }}$. To solve these differential equations, we need proper boundary conditions at the Earth's surface, the intersection to the ionosphere, and at the interior boundaries at the altitudes $z_{\text {low }}$ and $z_{\text {mid }}$.

\subsection{Lower boundary condition}

As the lower boundary condition, the vertical component of the electric field on the Earth's surface is given by

$-\left.\frac{\partial}{\partial z} \Phi(x, z)\right|_{z=0}=E_{0}(x)$.

Detailed measurements of this function are not available. Therefore, we use only typical values, presented in Rycroft et al. (2008) and other papers.

In the previous model from Denisenko et al. (2008) we followed the models similar to Pulinets et al. (1998) and Boyarchuk et al. (2004) and analyzed $E_{0}(x)$ negative in the domain of interest and zero outside of it. A negative vertical electric field $E_{0}(x)$, in accordance with Ohm's law, means a current from the atmosphere to ground. In view of the charge conservation law, somewhere exists an opposite current, and it is at infinity in such a unipolar model. When we analyze an earthquake preparation zone, the whole physical process is a

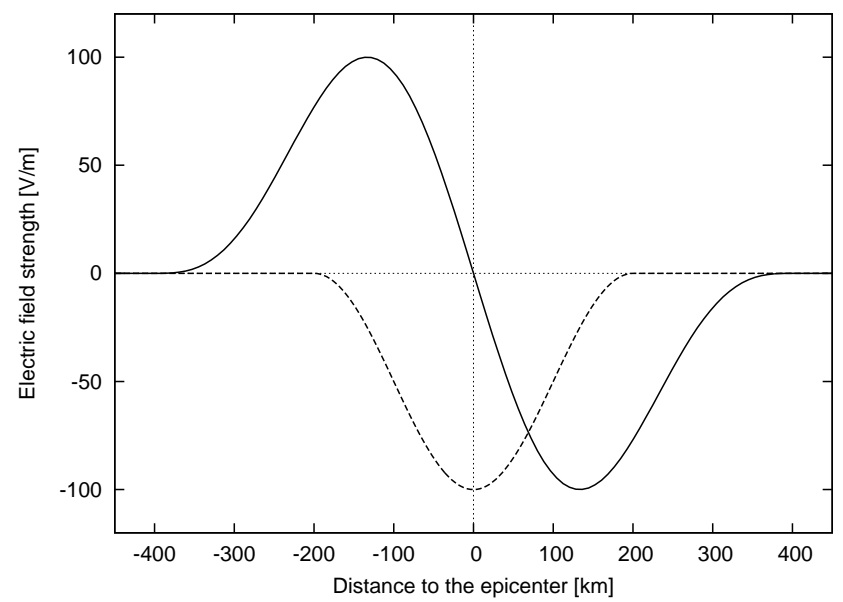

Fig. 2. Vertical electric field $E_{z}(x, 0)$ distribution at the Earth's surface. The curves correspond to the bipolar electric field as considered here (solid line), and the previous model by Denisenko et al. (2008) (dashed line). Both maximum values are $100 \mathrm{~V} \mathrm{~m}^{-1}$.

local one and both poles of the underground generator are in this region. To consider such a property, a bipolar $E_{0}(x)$ distribution is necessary. Here we use an anti-symmetric function that is the simplest among the bipolar ones. It is similar to that, used in the model by Kim et al. (1996).

The model distribution for the vertical electric field is a bipolar sin-distribution and can be written as (see Fig. 2),

$E_{0}(x)=-\bar{E}_{0} \gamma\left[1+\cos \left(\frac{x \pi}{a}\right)\right] \sin \left(\frac{x \pi}{a}\right),|x|<a$,

where $a$ indicates the size of the affected area on the Earth's surface, so that $E_{0}(x)=0$ outside, $\bar{E}_{0}$ is the maximal value of the electric field at $z=0$, and $\gamma$ is a normalization factor that makes the multiplier after $\bar{E}_{0}$ is restricted by unit. Quantity $a$ can be interpreted as the earthquake preparation area. The function $E_{0}(x)$ reachs its maximal value $\bar{E}_{0}$ at $x=a / 3$. It can be seen in Fig.2, that shows $E_{0}(x)$ with $a=400 \mathrm{~km}$.

Direct measurements of the electric field on the Earth's surface show that disturbances occur before earthquakes in the epicentral area (Kim et al., 1995). The amplitudes of those disturbances have been found to range from several tens up to $100-150 \mathrm{~V} \mathrm{~m}^{-1}$, before weak earthquakes (Kondo, 1968), and from several hundreds up to $1000 \mathrm{~V} \mathrm{~m}^{-1}$ before strong earthquakes (Vershinin et al., 1999). We use $100 \mathrm{~V} \mathrm{~m}^{-1}$ for a moderate earthquake.

\subsection{Interior boundary condition}

Since we have a vertical profile of the electric conductivity which is separated at altitudes $z=z_{\text {low }}$ and $z=z_{\text {mid }}$ in three regions near the Earth's surface, the main part of the atmosphere and the upper atmosphere, we need some relations at these interior boundaries. 
In view of the charge conservation law (Eq. 2), the vertical component $j_{\mathrm{Z}}$ must be continuous,

$j_{\mathrm{z}}\left(z_{\text {low }, \text { mid }}+\varepsilon\right)=j_{\mathrm{z}}\left(z_{\text {low }, \text { mid }}-\varepsilon\right)$,

where $\varepsilon \rightarrow 0$ means the limits from the domain $z>z_{\text {low }}$ and from $z<z_{\text {low }}$ when $\varepsilon>0$ and $\varepsilon<0$, respectively. The same is also near $z=z_{\text {mid }}$.

Since $j_{\mathrm{z}}=\sigma E_{\mathrm{z}}$ and the function $\sigma$ has no jump at $z_{\text {low }}$ and $z_{\text {mid }}$, from (Eq. 12) we obtain

$E_{\mathrm{Z}}\left(z_{\text {low }, \text { mid }}+\varepsilon\right)=E_{\mathrm{Z}}\left(z_{\text {low }, \text { mid }}-\varepsilon\right)$.

As a consequence of Eq. (1) the horizontal component $E_{\mathrm{x}}$ must be continuous,

$E_{\mathrm{X}}\left(z_{\text {low }, \text { mid }}+\varepsilon\right)=E_{\mathrm{X}}\left(z_{\text {low }, \text { mid }}-\varepsilon\right)$.

Since the electric field can be derived from the electric potential $\Phi$ in accordance to (Eq. 4), the interior boundary conditions (Eqs. 13 and 14) are equivalent to the continuity of the electric potential $\Phi(x, z)$ and the continuity of its vertical derivative,

$$
\begin{gathered}
\left.\Phi(x, z)\right|_{z=z_{\text {low }, \text { mid }}+\varepsilon}=\left.\Phi(x, z)\right|_{z=z_{\text {low }, \text { mid }}-\varepsilon}, \\
\left.\frac{\partial \Phi(x, z)}{\partial z}\right|_{z=z_{\text {low }, \text { mid }}+\varepsilon}=\left.\frac{\partial \Phi(x, z)}{\partial z}\right|_{z=z_{\text {low }, \text { mid }}-\varepsilon} .
\end{gathered}
$$

Since there is no difference between the interior boundaries, Eqs. (15) and (16) are valid for both intersections, at altitudes of $z=z_{\text {low }}$ and $z=z_{\text {mid }}$.

\subsection{Upper boundary condition}

The big differences in the results of several investigations for the penetration of an electric field into the ionosphere are mainly related to the upper boundary condition (Grimalsky et al., 2003). For this model, we use the relation which was obtained by Denisenko et al. (2008).

In our model we assumed straight and vertical geomagnetic field lines, what is nearly fulfilled in polar regions. Because of the nearly infinite field-aligned conductivity in the ionosphere, our model is not sensitive to the value of $z_{\text {up }}$. Test calculations show no significant change if $z_{\text {up }}$ is chosen in the range $80-90 \mathrm{~km}$ and in accordance to Denisenko et al. (2008), we apply $z_{\mathrm{up}}=80 \mathrm{~km}$. Namely, the horizontal electric field $E_{x}$ in the ionosphere, that is the main result parameter of the model, would be $0,2 \%$ less if we use $z_{\text {up }}=90 \mathrm{~km}$ and continue the scalar conductivity distribution (Eq. 7) till this height.

Because of the huge conductivity along the geomagnetic field lines, they can be considered as equipotential and the electric field normal to the magnetic field is independent of the height for $z>z_{\text {up }}$. Therefore, Ohm's law can be integrated over $z$ and when we take into account the charge conservation law at the altitude $z=z_{\text {up }}$, we can write the upper boundary condition for equation (Eq. 9)

$\left.\left\{-\frac{\partial}{\partial x}\left(\Sigma_{\mathrm{P}} \frac{\partial \Phi}{\partial x}\right)+\sigma\left(z_{\text {up }}\right) \frac{\partial \Phi}{\partial z}\right\}\right|_{z=z_{\text {up }}}=0$,

where $\Sigma_{\mathrm{P}}$ is the integrated Pedersen conductivity, that is always introduced in large scale ionospheric models (Hargreaves, 1979). We use just a constant $\Sigma_{\mathrm{P}}$, since the horizontal scale of interest is much less than the horizontal scale of the ionosphere.

\section{Model calculation}

To check out the influence of ionospheric conductivity variations on the electric field penetration through the atmosphere, we use the functional characteristics for the initial values of the conductivity $\bar{\sigma}$ and the scale height $h$ for the three different layers (Eq. 7). This relation is approximated to the empirical model presented by Molchanov and Hayakawa (2008), as it is shown in Fig. 1, and consider different values of $\Sigma_{\mathrm{P}}$. We use the values for the integrated conductivity $\Sigma_{\mathrm{P}}$ which are given by $\Sigma_{\mathrm{P}}=10 \mathrm{~S}$ in day-time (and in auroral zone) and $\Sigma_{\mathrm{P}}=0.1 \mathrm{~S}$ in night-time, respectively.

The boundary value problem (Eqs. 9, 10 and 15-17) aught be solved in the two-dimensional domain $0<z<z_{\text {up }}$, that is infinite in $\mathrm{x}$-direction. Acknowledging the fact that it is much more simple to deal with periodic functions, we present the distribution (Eq. 11) with Fourier series. For this aim, we preliminary continue the function over the whole $\mathrm{x}$-axis with a period of $2 b$, where we chose the parameter $b \approx 10 \cdot a$ and $b \gg a$ in such a manner, that the calculations in the domain of interest, $|x|<2 a$, are not disturbed by this modification.

We designed a new function $E_{1}(x)$ which is equal to $E_{0}(x)$ in the interval $|x|<b-a$ and

$E_{1}(x+b)=-E_{1}(x)$.

When we present this periodic function in a Fourier series, this leads to

$$
\begin{aligned}
E_{1}(x) & =\sum_{n=1}^{\infty} g_{n} \sin \left(k_{n} x\right), \\
g_{n} & =\frac{1}{b} \int_{0}^{2 b} E_{1}(x) \sin \left(k_{n} x\right) d x,
\end{aligned}
$$

where $k_{n}=(2 n-1) \pi / b$ and $g_{n}$ are the Fourier coefficients. The terms with $\cos \left(k_{n} x\right)$ as well as all terms with even values $2 n$ are absent, because the function $E_{1}(x)$ is antisymmetric with respect to $x=0, b, 2 b, \ldots$ and symmetric for $x=b / 2,3 b / 2, \ldots$.

The interval of integration in (Eq. 20) may be decreased to $0<x<a$, since $E_{1}(x)$ is antisymmetric and $E_{1}(x)=0$ at $a<x<b / 2$. Only the interval $0<x<a$ may be used. Because of that, $E_{1}(x)=E_{0}(x)$ at $0<x<a$ the expression 
(Eq. 11) may be used and the integral can be calculated analytically,

$$
\begin{aligned}
& g_{n}=-\frac{2 \bar{E}_{0} \gamma}{b} \int_{0}^{a}\left[1+\cos \left(\frac{x \pi}{a}\right)\right] \sin \left(\frac{x \pi}{a}\right) \sin \left(k_{n} x\right) d x \\
& =-6 a \bar{E}_{0} \gamma \sin \left(k_{n} a\right) /\left(b \pi\left[4-5\left(\frac{k_{n} a}{\pi}\right)^{2}+\left(\frac{k_{n} a}{\pi}\right)^{4}\right]\right) .
\end{aligned}
$$

The particular solution of Eq. (9) for each layer can be found due to separation of variables and is a superposition of the functions, depending on $x$ and $z$ separately. The general solution can be presented as a sum of the particular solutions with arbitrary coefficients,

$\Phi(x, z)=\sum_{n=1}^{\infty} \sin \left(k_{n} x\right) \Psi_{n}(z)$,

where

$\Psi_{n}(z)=\left[E_{n} \exp \left(\lambda_{5 n} z\right)+F_{n} \exp \left(\lambda_{6 n} z\right)\right], z_{\text {mid }} \leq z \leq z_{\text {up }}$,

$\Psi_{n}(z)=\left[C_{n} \exp \left(\lambda_{3 n} z\right)+D_{n} \exp \left(\lambda_{4 n} z\right)\right], z_{\text {low }} \leq z<z_{\text {mid }}$

$\Psi_{n}(z)=\left[A_{n} \exp \left(\lambda_{1 n} z\right)+B_{n} \exp \left(\lambda_{2 n} z\right)\right], \quad 0 \leq z<z_{\text {low }}$,

with

$\lambda_{5 n, 6 n}=-\frac{1}{2 h_{3}} \pm \sqrt{\frac{1}{4 h_{3}^{2}}+k_{n}^{2}}$,

$\lambda_{3 n, 4 n}=-\frac{1}{2 h_{2}} \pm \sqrt{\frac{1}{4 h_{2}^{2}}+k_{n}^{2}}$,

$\lambda_{1 n, 2 n}=-\frac{1}{2 h_{1}} \pm \sqrt{\frac{1}{4 h_{1}^{2}}+k_{n}^{2}}$.

The $\mathrm{x}$ - and $\mathrm{z}$-components of the electric field can be calculated by differentiation of the series (Eq. 22). When we put them into the conditions (Eqs. 10 and 15-17), we obtain equalities of the Fourier series's. Since the functions $\sin \left(k_{n} x\right)$ are orthogonal ones, each of them leads to an infinite set of equalities for each $n$ separately.

For each index $n$ we derive a separate system of six linear algebraic equations where the coefficients $A_{n}, B_{n}, C_{n}, D_{n}$, $E_{n}$, and $F_{n}$ are the unknowns. The only non-zero right hand side exists in the equation derived from (Eq. 10) and equals to the already known values $g_{n}$ (Eq. 20),

$\Gamma \cdot\left(\begin{array}{c}A_{n} \\ B_{n} \\ C_{n} \\ D_{n} \\ E_{n} \\ F_{n}\end{array}\right)=\left(\begin{array}{c}-g_{n} \\ 0 \\ 0 \\ 0 \\ 0 \\ 0\end{array}\right)$

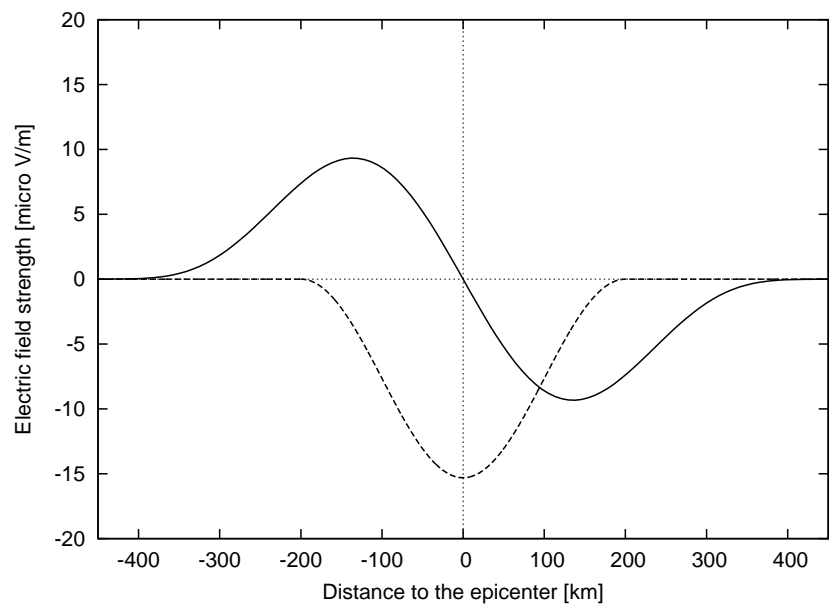

Fig. 3. Vertical electric field at the bottom of the ionosphere $E_{\mathrm{Z}}\left(x, z_{\text {up }}\right)$ is compared to the model $E_{\mathrm{Z}}\left(x, z_{\mathrm{up}}\right) / 10$ (Denisenko et al., 2008) (solid and dashed lines), with maximum values of $9.3 \mu \mathrm{V} \mathrm{m}^{-1}$ and $153 \mu \mathrm{V} \mathrm{m}^{-1}$, respectively.

where the matrix $\Gamma$ and the coefficients are

$\Gamma=\left(\begin{array}{cccccc}\lambda_{1 n} & \lambda_{2 n} & 0 & 0 & 0 & 0 \\ \alpha_{1} & \alpha_{2} & -\alpha_{3} & -\alpha_{4} & 0 & 0 \\ \lambda_{1 n} \alpha_{1} & \lambda_{2 n} \alpha_{2} & -\lambda_{3 n} \alpha_{3} & -\lambda_{4 n} \alpha_{4} & 0 & 0 \\ 0 & 0 & \alpha_{5} & \alpha_{6} & -\alpha_{7} & -\alpha_{8} \\ 0 & 0 & \lambda_{3 n} \alpha_{5} & \lambda_{4 n} \alpha_{6} & -\lambda_{5 n} \alpha_{7} & -\lambda_{6 n} \alpha_{8} \\ 0 & 0 & 0 & 0 & \beta_{1} & \beta_{2}\end{array}\right)$,

$\alpha_{1}=\exp \left(\lambda_{1 n} z_{\text {low }}\right), \quad \alpha_{2}=\exp \left(\lambda_{2 n} z_{\text {low }}\right)$,

$\alpha_{3}=\exp \left(\lambda_{3 n} z_{\text {low }}\right), \quad \alpha_{4}=\exp \left(\lambda_{4 n} z_{\text {low }}\right)$,

$\alpha_{5}=\exp \left(\lambda_{3 n} z_{\text {mid }}\right), \quad \alpha_{6}=\exp \left(\lambda_{4 n} z_{\text {mid }}\right)$,

$\alpha_{7}=\exp \left(\lambda_{5 n} z_{\mathrm{mid}}\right), \quad \alpha_{8}=\exp \left(\lambda_{6 n} z_{\mathrm{mid}}\right)$,

$\beta_{1}=\left(\Sigma_{P} k_{n}^{2}+\sigma\left(z_{\text {up }}\right) \lambda_{5 n}\right) \exp \left(\lambda_{5 n} z_{\text {up }}\right)$,

$\beta_{2}=\left(\Sigma_{P} k_{n}^{2}+\sigma\left(z_{\mathrm{up}}\right) \lambda_{6 n}\right) \exp \left(\lambda_{6 n} z_{\mathrm{up}}\right)$.

Now the two components of the electric field in the atmosphere can be calculated from Eq. (4)

$E_{x}(x, z)=-\frac{\partial}{\partial x} \Phi(x, z)$,

$E_{z}(x, z)=-\frac{\partial}{\partial z} \Phi(x, z)$,

where the potential $\Phi(x, z)$ is calculated by formulas (Eqs. 22-25). The third component $E_{\mathrm{y}}(x, z)=0$, since there is no variation along the $y$-coordinate.

\section{Summary of the main results}

The results of this model for the electric field calculation in the Earth's atmosphere for the night-time ionosphere are shown in Figs. 3-7. 


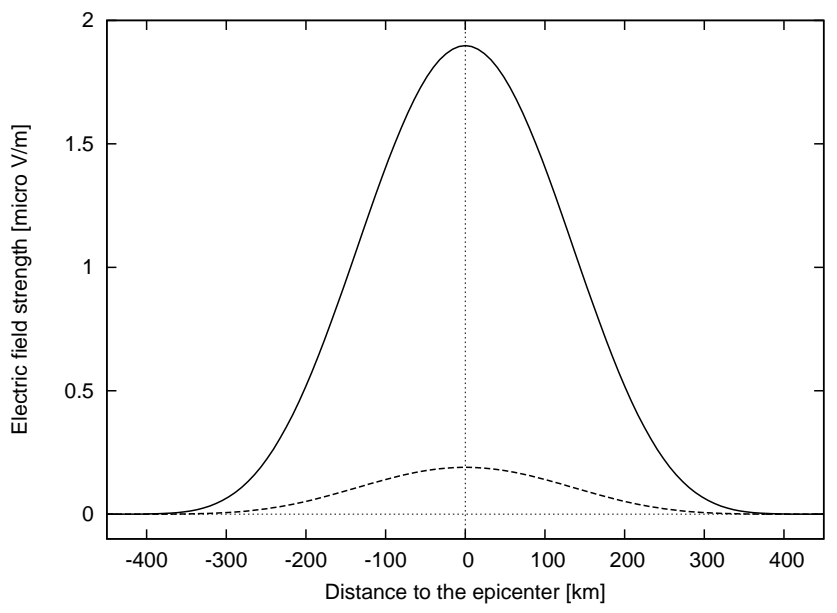

Fig. 4. Horizontal electric field $E_{\mathrm{X}}\left(x, z_{\mathrm{up}}\right)$ in the night-time ionosphere with $\Sigma_{\mathrm{P}}=0.1 S$ (solid line) and $\Sigma_{\mathrm{P}}=1 S$ (dashed line).

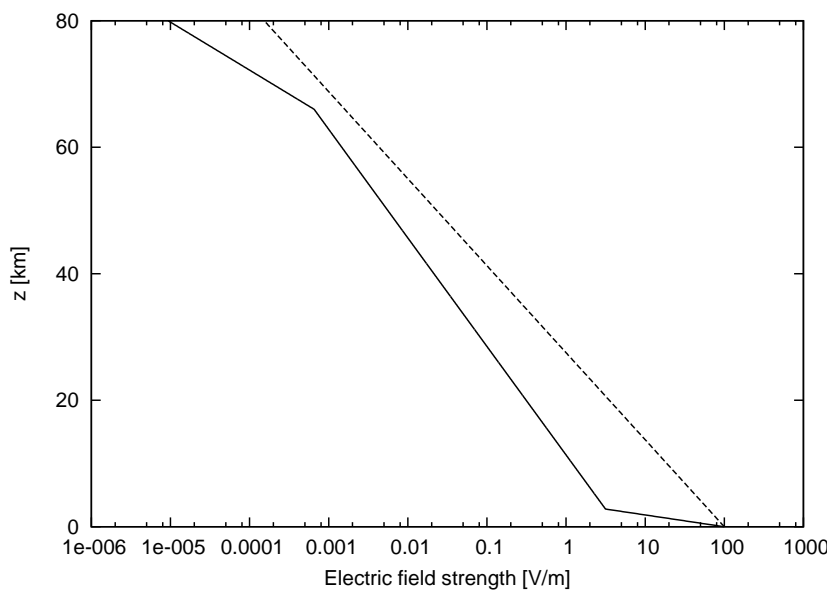

Fig. 5. Height distributions of the maximum values for the vertical electric field $E_{\mathrm{Z}}(a / 3, z)$ in this model (solid line) and $E_{\mathrm{Z}}(0, z)$ in the model of Denisenko et al. (2008) (dashed line).

The shape of the graphs of the vertical electric field $E_{\mathrm{Z}}(x, z)$ is almost independent of the altitude as it can be seen by comparison of Figs. 3 and 2. The $E_{\mathrm{z}}(x, z)$ distributions at the ground and at the upper boundary of the atmosphere are plotted in different scales, where the maximum values are equal to $100 \mathrm{~V} \mathrm{~m}^{-1}$ at ground and $9.3 \mu \mathrm{V} \mathrm{m}^{-1}$ at $z=z_{\text {up. The }}$ shape of the graphs of the horizontal electric field $E_{\mathrm{x}}(x, z)$ stays also about the same at different $z=$ const and is shown in Fig. 4. The height distributions of $E_{\mathrm{x}}(x, z)$ and $E_{\mathrm{z}}(x, z)$ can be presented with their maximal values which are obtained at $x=0$ and $x=a / 3$, respectively. These maximal values are shown in Figs. 5 and 6 as functions of $z$.

Since we have chosen a bipolar distribution for the electric field we calculate the penetration into the ionosphere with a

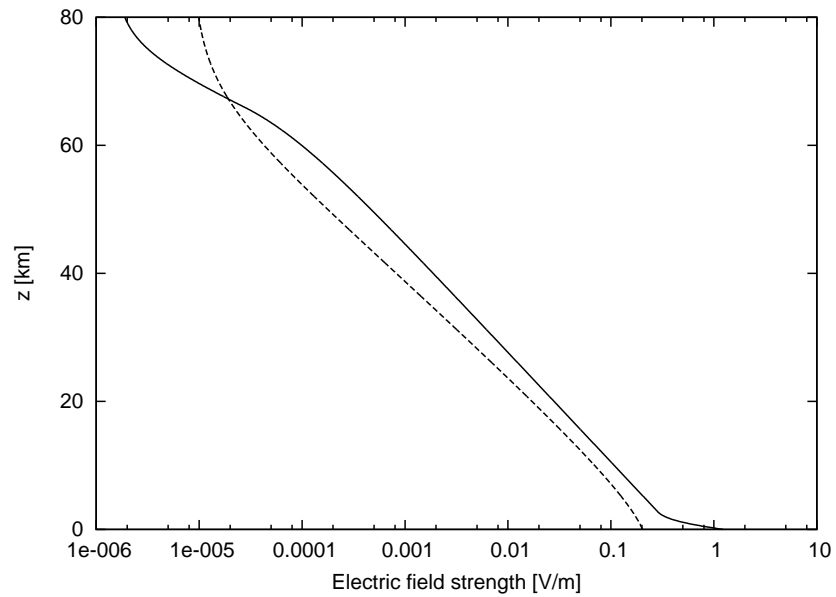

Fig. 6. Height distributions of the maximum values for the horizontal electric field $E_{\mathrm{X}}(0, z)$ in this model (solid line) and $E_{\mathrm{X}}(a, z)$ in the model of Denisenko et al. (2008) (dashed line).

characteristic source size dimension of $a=400 \mathrm{~km}$, which is twice the size considered by Denisenko et al. (2008). This is necessary to stay clear that the same total electric field flux,

$\int_{0}^{a} E_{\mathrm{Z}}(x, 0) d x$,

is going upward from the ground. Such a common parameter is useful for comparison of the results of bipolar and unipolar models.

The horizontal electric field $E_{\mathrm{x}}(x, z)$ at the altitude of $80 \mathrm{~km}$ is shown in Fig. 4 and it stays the same in the ionosphere for $z>80 \mathrm{~km}$ because of the large field-aligned conductivity. It is inverse proportional to the integrated Pedersen conductivity $\Sigma_{\mathrm{P}}$. The typical value of the day-time ionospheric Pedersen conductance is about 100 times higher than during night-time. Figure 4 shows the horizontal electric field for night-time conditions with $\Sigma_{\mathrm{P}}=0.1 \mathrm{~S}$. The maximum value of $E_{\mathrm{x}}\left(x, z_{\mathrm{up}}\right)$ equals to $1.9 \mu \mathrm{V} \mathrm{m}^{-1}$. The daytime $E_{\mathrm{X}}(x, z)$ distribution has almost the same shape, but it is invisible in this scale because its maximum equals about $0.02 \mu \mathrm{V} \mathrm{m}^{-1}$.

The electric fields of these magnitudes cannot be observed in the ionosphere because much larger fields of other origin are always present there.

We investigate the relation between the source size dimension and the share of the current which is going up into the ionosphere. For this analysis, we design the current function $\mathbf{C}$ which can be derived from the relation

$\mathbf{j}=\nabla \times \mathbf{C}$,

that means

$j_{x}=-\frac{\partial C}{\partial z}$, 


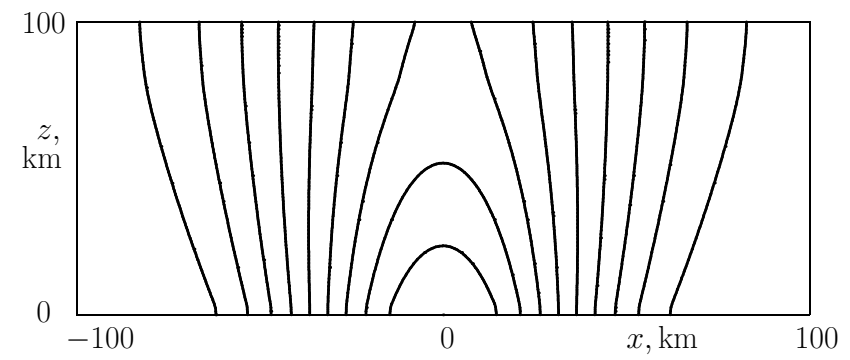

Fig. 7. Current lines for a source size dimension of $a=100 \mathrm{~km}$, where about $30 \%$ of the total current is closed inside the atmosphere.

$j_{z}=\frac{\partial C}{\partial x}$

where $C(x, z)$ is the y-component of the function $\mathbf{C}$ which $\mathrm{X}-, \mathrm{Z}$-components equals zero.

Since an arbitrary constant can be added to $C$ with no change of (Eqs. 33 and 34), we can put $C(-b, z)=0$. Because of symmetry, we have $j_{\mathrm{x}}=0$ at the vertical line $x=-b$ as well as at $x=b$. Therefore, we can integrate (Eq. 33) over $z$ at $x=-b$ and obtain $C(-b, z)=0$. Now we can integrate (Eq. 34) at each level $z=$ const and construct

$C(x, z)=\int_{-b}^{x} j_{\mathrm{Z}}\left(x^{\prime}, z\right) d x^{\prime}$.

In accordance to Ohm's law (Eq. 3), the definition of the electric potential (Eq. 4), and the solution for the problem as the Fourier series's (Eqs. 22-25), we can express $j_{\mathrm{z}}(x, z)$ as the Fourier series with already known coefficients and integrate each term separately at each level $z=$ const inside each of three layers in the atmosphere

$$
\begin{array}{lr}
C_{1 n}(x, z)=-\sigma(z) \omega_{1} / k_{n}, & 0 \leq z<z_{\text {low }}, \\
C_{2 n}(x, z)=-\sigma(z) \omega_{2} / k_{n}, & z_{\text {low }} \leq z<z_{\text {mid }}, \\
C_{3 n}(x, z)=-\sigma(z) \omega_{3} / k_{n}, & z_{\text {mid }} \leq z \leq z_{\text {up }},
\end{array}
$$

where

$$
\begin{aligned}
& \omega_{1}=\left[A_{n} \lambda_{1 n} \exp \left(\lambda_{1 n} z\right)+B_{n} \lambda_{2 n} \exp \left(\lambda_{2 n} z\right)\right] \cos \left(k_{n} x\right), \\
& \omega_{2}=\left[C_{n} \lambda_{3 n} \exp \left(\lambda_{3 n} z\right)+D_{n} \lambda_{4 n} \exp \left(\lambda_{4 n} z\right)\right] \cos \left(k_{n} x\right), \\
& \omega_{3}=\left[E_{n} \lambda_{5 n} \exp \left(\lambda_{5 n} z\right)+F_{n} \lambda_{6 n} \exp \left(\lambda_{6 n} z\right)\right] \cos \left(k_{n} x\right) .
\end{aligned}
$$

The resulting current functions are presented in Figs. 7 and 8 for two values of the horizontal scale of the electric field source at ground, $a=100 \mathrm{~km}$ and $a=10 \mathrm{~km}$. These values are typical for a large scale source whose current is closed in the ionosphere and for a small scale source with closure being mainly in the atmosphere. Calculations show that only

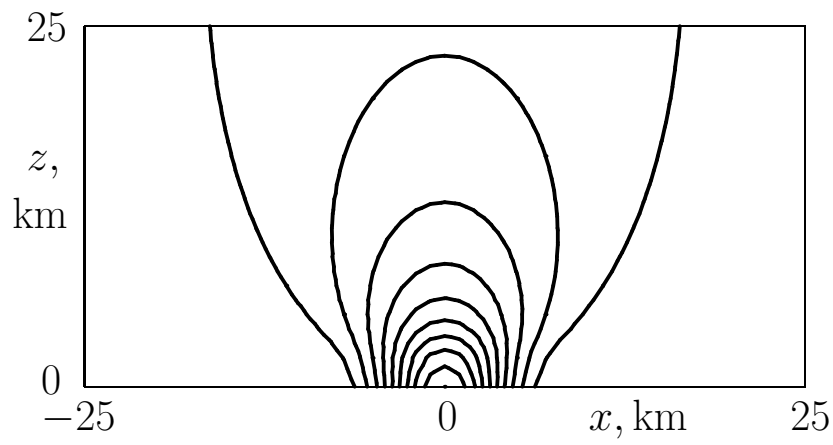

Fig. 8. Current lines for a source size dimension of $a=10 \mathrm{~km}$.

about $3 \%$ of the total vertical current is closed inside the atmosphere for $a=400 \mathrm{~km}$ and about $30 \%$ for $a=100 \mathrm{~km}$ (Fig. 7). For a source size dimension of $a=10 \mathrm{~km}$, the horizontal resistivity is much less than the vertical one and only about $20 \%$ of current reaches ionospheric heights (Fig. 8). The total currents are $2 \times 10^{-7} \mathrm{~A} \mathrm{~m}^{-1}$ and $5 \times 10^{-9} \mathrm{~A} \mathrm{~m}^{-1}$ for a characteristic source size of $a=400 \mathrm{~km}$ and $a=10 \mathrm{~km}$, respectively. The distance between the current lines in Figs. 7 and 8 are a tenth of the total currents.

In accordance to definition (Eqs. 33 and 34), the lines at which $C(x, z)=$ const are the current lines for $\mathbf{j}(x, z)$. In our model as well as in each two-dimensional model, we define the current function for the layer $\delta y=1 \mathrm{~m}$, and so the current is measured in $\mathrm{A} \mathrm{m}^{-1}$ instead of Amperes, or we would say that this amount of Amperes exists in a $\delta y=1 \mathrm{~m}$ layer.

\section{Discussion and conclusion}

The values of the horizontal electric field $E_{\mathrm{X}}(x, z)$ at the bottom of the night-time ionosphere are in the $\mu \mathrm{V} \mathrm{m}^{-1}$ range and about 100 times less during the day. As compared to the results of Denisenko et al. (2008), the electric field penetration from the lithosphere into the ionosphere is much weaker. The maximum value of the vertical component of the electric field at an altitude of $z=80 \mathrm{~km}$ is about 15 times less than in the previous model. It can be simply explained by the modification of $\sigma(z)$ that is done in this model (see Fig. 1). It can be seen in Fig. 1, that $\sigma(0)$ has become ten times less. Since the total electric field flux (Eq. 31) is left about the same, the total current has become 10 times less. This vertical current stays approximately the same at all heights in the atmosphere. It can be seen from Fig. 1 that $\sigma\left(z_{\text {up }}\right)$ has become about 1.6 times larger. The same current would be provided by 1.6 times smaller $E_{\mathrm{z}}\left(x, z_{\mathrm{up}}\right)$, and 10 times less current by about 16 times less $E_{\mathrm{z}}\left(x, z_{\text {up }}\right)$. We have just this result in our new model.

In the model of Denisenko et al. (2008), the horizontal electric field $E_{\mathrm{X}}\left(x, z_{\mathrm{up}}\right)$ of about $10 \mu \mathrm{V} \mathrm{m}^{-1}$ penetrating into the night-time ionosphere, was obtained. Here we have 5 
times less for the vertical electric field at ground on the same scale. As mentioned above, we have saved the same electric field flux at ground (Eq. 31) and have made ten times less total vertical current. This amount is closed by the ionospheric current. For the same ionospheric integrated conductivity it would be provided by a ten times smaller $E_{\mathrm{x}}\left(x, z_{\text {up }}\right)$. The previous model was an unipolar distribution and so this current was divided to both directions $x \rightarrow+\infty$ and $x \rightarrow-\infty$ in contrast with the only direction from left to right in this model. That is why we obtain here $1.9 \mu \mathrm{V} \mathrm{m}^{-1}$ near $x=0$ instead of $\pm 10 \mu \mathrm{V} \mathrm{m}^{-1}$ at $x \rightarrow \pm \infty$ in that model.

In accordance to Ohm's law, the total vertical electric current $I$ near ground is equal to the total electric field flux (Eq. 31) multiplied by $\sigma(0)$. When the horizontal scale $a$ exceeds $100 \mathrm{~km}$, almost the whole current $I$ goes from ground to the ionosphere as can be seen in Fig. 7, where it is closed. Hence the ionospheric electric current almost equals to $I$ and the appropriate $E_{\mathrm{x}}$ is about $I / \Sigma_{\mathrm{P}}$. Therefore, the maximal value of $E_{\mathrm{x}}$ which penetrates into the ionosphere is not sensitive to details of the conductivity height distribution defined by the parameters (Eq. 7), and only $\sigma(0)$ is significant when $a>100 \mathrm{~km}$. If $a \ll 100 \mathrm{~km}$ other parameters (Eq. 7) are important because they define the atmospheric part of the closure current.

Because of the assumption that geomagnetic field lines are parallel in the ionosphere and the field-aligned conductivity is huge, the electric field strength does not vary significantly in higher altitudes.

The main critics is related to the simplified usage of an isotropic electric conductivity. Our approximation for the empirical conductivity profile (Molchanov and Hayakawa, 2008) is rather good as it can be seen from Fig. 1. In the main aim to get analytically solutions, the approximation function is not smooth at the interior boundaries.

As shown in Fig. 5, the vertical electric field $E_{\mathrm{z}}$ quickly decreases with height in contrast with $E_{\mathrm{x}}$ which slowly varies in the higher atmosphere similar to that it stays constant in the ionosphere (see Fig. 6). We think that the parameter of interest in our model, which is $E_{\mathrm{X}}$ in the ionosphere, is not sensitive to the discussed simplification of a real conductivity profile.

However, the conclusion for this model distribution is that the penetration of the electric field of lithospheric origin into the ionosphere is too weak for a measurable result at ionospheric heights, due to the presence of larger electric fields of other nature. The typical magnitude of the electric field in the middle latitude ionosphere is a few $\mathrm{mV} \mathrm{m}^{-1}$. To make the maximal value of the ionospheric electric field penetrated from ground equal to $2 \mathrm{mV} \mathrm{m}^{-1}$ instead of obtained $2 \mu \mathrm{V} \mathrm{m}^{-1}$ the vertical electric field near ground aught be proportionally larger, $100 \mathrm{kV} \mathrm{m}^{-1}$ instead of $100 \mathrm{~V} \mathrm{~m}^{-1}$, since the model is a linear one. In accordance to Rycroft et al. (2008), such a huge value is never observed. Vershinin et al. (1999) report about electric fields up to $1 \mathrm{kV} \mathrm{m}^{-1}$ before strong earthquakes, that is hundred times less than necessary.
The conductivity near ground $\sigma(0)$ can be in the range $10^{-14}-10^{-13} \mathrm{~S} \mathrm{~m}^{-1}$ as it is shown in the handbook by Campen et al. (1960). The last value of $\sigma(0)$ induces that the electric field in the ionosphere is about ten times larger than in the actual model. It is comparable to the model by Denisenko et al. (2008) and corresponds to the absence of the near ground layer with low conductivity in that model as it can be seen in Fig. 1. Hence, $10 \mathrm{kV} \mathrm{m}^{-1}$ vertical electric field above ground would be enough to produce a few $\mathrm{mV} \mathrm{m}^{-1}$ horizontal electric field in the ionosphere.

Of course this does not mean that no other physical processes can produce earthquake precursors in the ionosphere. For example, Molchanov et al. (1995) have shown that electromagnetic waves in the frequency range $10^{-2}$ to $10^{2} \mathrm{~Hz}$ can penetrate from ground to the magnetosphere as Alfvén waves, which can be observed by satellites.

In future investigations, it is necessary to include the narrowness of the fault area in the y-direction as well as to provide a better approximation of the realistic conductivity profile in the atmosphere. This will require a numerical method to solve the boundary value problem for the electric potential in contrast to pure analytics as done here, which makes this model well suitable for verification. The anisotropic conductivity tensor at ionospheric heights also aught be taken into account, as it was discussed by Denisenko et al. (2008) as well as the role of inclination of the magnetic field lines.

Acknowledgements. This work is supported by grants 07-0500135, 09-06-91000 from the Russian Foundation for Basic Research and by the Program 16.3 of the Russian Academy of Sciences. Additional support is due to the Austrian "Fonds zur Förderung der wissenschaftlichen Forschung" under Project I193N16 and the "Verwaltungsstelle für Auslandsbeziehungen" of the Austrian Academy of Sciences.

The authors are grateful to the referees whose comments helped considerably to improve the paper.

Topical Editor K. Kauristie thanks two anonymous referees for their help in evaluating this paper.

\section{References}

Berthelier, J. J., Godefroy, M., Leblanc, F., Malingre, M., Menvielle, M., Lagoutte, D., Brochot, J. Y., Colin, F., Elie, F., Legendre, C., Zamora, P., Benoist, D., Chapuis, Y., Artru, J., and Pfaff, R.: ICE, the electric field experiment on DEMETER, Planet. Space Sci., 54, 456-471, 2006.

Boyarchuk, K. A., Lomonosov, A. M., Pulinets, S. A., and Hegai, V. V.: Variability of Earth's atmospheric electric field and ionaerosols kinetics in the troposphere, Studia Geophysicae et Geodaetica, 42, 197-210, 1998.

Campen, C. F., Ripley, W. S., Cole, A. E., Sissenwine, N., Condron, T. P., and Solomon, I.: Handbook of Geophysics, The Macmillan Company, New York, 1960.

Denisenko, V. V., Boudjada, M. Y., Horn, M., Pomozov, E. V., Biernat, H. K., Schwingenschuh, K., Lammer, H., Prattes, G., and Cristea, E.: Ionospheric conductivity effects on electrostatic field penetration into the ionosphere, Nat. Hazards Earth Syst. Sci., 8, 
1009-1017, 2008,

http://www.nat-hazards-earth-syst-sci.net/8/1009/2008/.

Debuev, V. and Zelenova, T.: Electron density profile changes in a pre-earthquake period, Adv. Space Res., 18(6), 115-118, 1996.

Gokhberg, M. B., Gufel'd, I. L., Gershenzon, N. I., and Pilipenko, V. A.: Electromagnetic effects during rupture of the Earth's crust, Izvestiya Russian Academy of Sciences, Phys. Solid Earth, 21, 52-63, 1985.

Gokhberg, M. B., Pilipenko, V. A., and Pokhotelov, O. A.: Seismic precursors in the ionosphere, Izvestiya Russian Academy of Sciences, Phys. Solid Earth, 19(10), 762-765, 1983.

Hargreaves, J. K.: The upper atmosphere and Solar-Terrestrial relations, Van Nostrand Reinold Co. Ltd, NY, 1979.

Kelley, M. C., Livingston, R., and McCready, M.: Large amplitude thermospheric oscillations induced by an earthquake, Geophys. Res. Lett., 12, 577-580, 1985.

Kim, V. P., Khegaj, V. V., and Nikiforova, L. I.: On a possible distortion of the nighttime ionopheric E-region above large-scale tectonic fault, Phys. Solid Earth, 31(7), 580-584, 1996.

Kim, V. P., Hegaj, V. V., and Illich-Switych, P. V.: On the possibility of a metallic ion layer forming in the E-region of the night midlatitude ionosphere before great earthquakes, Geomagnetism and Aeronomy, 33, 658-662, 1994.

Kingsley, S. P.: On the possibilities for detecting radio emissions from earthquakes, Il Nuovo Cimento, 12, 117-120, 1989.

Kondo, G.: The variation of the atmospheric electric field at the time of earthquake, Memoirs of the Kakioka Magnetic Observatory, 13, 11-23, 1968.

Liu, J. Y., Chuo, Y. J., Shan, S. J., Tsai, Y. B., Chen, Y. I., Pulinets, S. A., and Yu, S. B.: Pre-earthquake ionospheric anomalies registered by continuous GPS TEC measurements, Ann. Geophys., 22, 1585-1593, 2004, http://www.ann-geophys.net/22/1585/2004/.

Molchanov, O. and Hayakawa, M.: Seismoelectromagnetics and related phenomena: History and latest results, Terrapub, Tokyo, Appendixes 9, 10, 2008.

Molchanov, O. A. and Hayakawa, M.: Generation of ULF electromagnetic emissions by microfracturing, Geophys. Res. Lett., 22, 3091-3094, 1995.

Molchanov, O. A., Hayakawa, M., and Rafalsky, V. A.: Penetration characteristics of electromagnetic emissions from an underground seismic source into the atmosphere, ionosphere and magnetosphere, J. Geophys. Res., 100(A2), 1691-1712, 1995.

Molchanov, O., Fedorov, E., Schekotov, A., Gordeev, E., Chebrov, V., Surkov, V., Rozhnoi, A., Andreevsky, S., Iudin, D., Yunga, S., Lutikov, A., Hayakawa, M., and Biagi, P. F.: Lithosphereatmosphere-ionosphere coupling as governing mechanism for preseismic short-term events in atmosphere and ionosphere, Nat. Hazards Earth Syst. Sci., 4, 757-767, 2004, http://www.nat-hazards-earth-syst-sci.net/4/757/2004/.
Park, C. G. and Dejnakarintra, M.: Penetration of thundercloud electric fields into the ionosphere and magnetosphere, 1. Middle and auroral latitudes, J. Geophys. Res., 84, 960-964, 1973.

Pogorel'tsev, A. I.: Disturbances of electric and magnetic fields induced by the interaction of atmospheric waves with the ionospheric plasma, Geomagnetism and Aeronomy, 29, 286-292, 1989 (in Russian).

Pulinets, S. A. and Boyarchuk, K.: Ionospheric precursors of earthquakes, Berlin Heidelberg New York: Springer, 2004.

Pulinets, S. A., Legen'ka, A. D., Gaivoronskaya, T. V., and Depuev, V. Kh.: Main phenomenological features of ionospheric precursors of strong earthquakes, J. Atmos. Solar-Terr. Phys., 65, 13371347, 2003.

Pulinets, S. A., Boyarchuk, K. A., Hegai, V. V., Kim, V. P., and Lomonosov, A. M.: Quasielectrostatic model of atmospherethermosphere-ionosphere coupling, Adv. Space Res., 26, 1209 1218, 2000.

Pulinets, S. A., Hegai, V. V., Kim, V. P., and Depuev, V. Kh.: Unusual longitude modification of the night-time mid-latitude F2 region ionosphere in July 1980 over the array of tectonic faults in the Andes area: Observations and interpretation, Geophys. Res. Lett., 25, 4133-4136, 1998.

Ruzhin, Yu. Ya. and Depueva, A. Kh.: Seismoprecursors in space as plasma and wave anomalies, J. Atmos. Electr., 16, 271-288, 1996.

Rycroft, M. J., Harrison, G. R., Nicoll, K. A., and Mareev, E. A.: An overview of Earth's global electric circuit and atmospheric conductivity, Space Sci. Rev., 137, 683-105, 2008.

Sharma, D. K., Rai, J., Chand, R., and Israil, M.: Effect of seismic activities on ion temperature in the $\mathrm{F} 2$ region of the ionosphere, Atmósfera, 19, 1-7, 2006.

Silina, A. S., Liperovskaya, E. V., Liperovsky, V. A., and Meister, C.-V.: Ionospheric phenomena before strong earthquakes, Nat. Hazards Earth Syst. Sci., 1, 113-118, 2001, http://www.nat-hazards-earth-syst-sci.net/1/113/2001/.

Sorokin, V. M., Chmyrev, V. M., and Yaschenko, A. K.: Electrodynamic model of the lower atmosphere and the ionosphere coupling, J. Atmos. Solar-Terr. Phys., 63, 1681-1691, 2001.

Vershinin, E. F., Buzevich, A. V., Yumoto, K., Saita, K., and Tanaka, Y.: Correlation of seismic activity with electromagnetic emissions and variations in Kamchatka region, Atmospheric and Ionospheric Electromagnetic Phenomena Associated with Earthquakes, Terrapub, Tokyo, 513-517, 1999. 